\title{
Alcohol-Induced Immune Dysregulation in the Colon Is Diurnally Variable
}

\author{
Eve Lauren Grumish Andrew R. Armstrong Robin M. Voigt \\ Christopher B. Forsyth Faraz Bishehsari \\ Division of Gastroenterology, Department of Internal Medicine, Rush University Medical Center, Chicago, IL, USA
}

\section{Keywords}

Alcohol · Colon cancer · Colon inflammation · Time · Circadian rhythm

\begin{abstract}
Introduction: Alcohol increases the risk of colon cancer. Colonic inflammation mediates the effects of alcohol on colon carcinogenesis. Circadian rhythm disruption enhances the alcohol's effect on colonic inflammation and cancer. Objective: Here, we investigate the diurnal variation of lymphocyte infiltration in the colonic mucosa in response to alcohol. Methods: Sixty C57BL6/J mice were fed a chow diet, and gavaged with alcohol at a specific time once per day for 3 consecutive days. Immunohistochemistry and immunofluorescence staining were used to quantify total, effector, and regulatory T cells in the colon. Student's $t$ test, one-way ANOVA, and two-way ANOVA were used to determine significance. Results: Following the alcohol binge, the composition of immune T cell subsets in the mouse colon was time-dependent. Alcohol did not alter the total number of $\mathrm{CD}^{+} \mathrm{T}$ cells. However, upon alcohol treatment, $\mathrm{T}$-bet ${ }^{+} \mathrm{T}$ helper 1 (Th1) cells appeared to dominate the $T$ cell population following a reduction in Foxp $3^{+}$regulatory $\mathrm{T}$ cell (Treg) numbers. Depletion of Tregs was time-dependent, and their numbers were dramatically reduced when alcohol was administered during the rest phase. A reduction in Tregs significantly increased the Th1/Treg ratio, resulting in a more proinflammatory milieu. Conclusions: Alcohol enhanced the proinflammatory profile in the colon mucosa, as demonstrated by a higher T-bet ${ }^{+} /$Foxp $^{+}{ }^{+}$ratio, especially during the rest phase. These findings may partly account for the interaction of circadian rhythm disruption with alcohol in colon inflammation and cancer.

(c) 2020 S. Karger AG, Basel
\end{abstract}

\section{Introduction}

Alcohol is an established risk factor for colon cancer (CRC) [1, 2]. The mechanisms of alcohol-induced CRC remains largely unknown, but accumulative evidence suggests that colonic inflammation mediates the effects of alcohol on colon carcinogenesis $[3,4]$. The composition of mucosal immune cells is important in regulating the colonic epithelial barrier. Impaired epithelial barrier function results in the passage of proinflammatory contents of the gut through the mucosa, leading to local and systemic inflammation and a predisposition to intestinal as well as extraintestinal alcohol-induced organ damage [5]. Inflammatory homeostasis in the colon is maintained by the suppressive activity of regulatory $\mathrm{T}$ cells (Tregs). Tregs are characterized by the expression of the transcription factor forkhead box P3 (Foxp3) and have been identified as key mediators in resolving tissue inflammation. Decreased Treg recruitment has been shown to be associated with dysregulated mucosal immunity and early steps of colon carcinogenicity [6-8]. Several chemokines are involved in Treg recruitment, which may be affected by alcohol [8]. Tregs exert their anti-inflammatory function by controlling the differentiation of naïve $\mathrm{T}$ cells into $\mathrm{T}$ helper 1 (Th1) cells [9]. T-bet ${ }^{+}$Th1 cells promote proinflammatory responses, recruit macrophages, and produce TNF- $\alpha$ and IFN- $\gamma[10,11]$. Factors that enhance alcohol-induced colonic inflammation may promote alcohol-induced CRC. Indeed, our group has previously shown that one such cofactor could be circadian rhythm disruption, which can exacerbate colonic inflammation in conjunction with alcohol [12-14]. Molecular clock is expressed within the adaptive immune system as well. 
Circadian rhythms have been reported in T cell responses [15], leukocyte trafficking [16], cell surface receptor expression, and cytokine and chemokine expression [1719]. In this study, we describe an abundance of total and subset $\mathrm{T}$ cell populations in the mouse colon following alcohol treatment to test the hypothesis that alcohol alters colonic $\mathrm{T}$ cell subset populations and that these alterations are time-dependent.

\section{Materials and Methods}

\section{Animal Model}

Male C57BL6/J mice (aged 6-8 weeks; Jackson Laboratories) were fed a standard chow diet (Teklad Envigo \#2018) for 4 weeks as described [20], within IACUC-approved, ventilated, light-tight cabinets. Mice were maintained on a constant 12-h light/dark cycle for the duration of the experiment. By convention, the time of light onset is referred to as Zeitgeber time (ZT) of zero i.e., ZT0, and lightsoff time is referred to as ZT12. All procedures conducted during the dark phase (i.e., ZT12-ZT0) were performed under red-light conditions. Mice were randomly assigned to receive either alcohol or vehicle control (PBS), following the NIAAA model of alcohol binge [21]. During the last week of the study, i.e., on days $43-45$, the mice received a total of 3 once-daily gavages of alcohol or PBS $(6 \mathrm{~g} / \mathrm{kg} /$ day) at the same time point each day. Each group $(n=5)$ received the binge treatment at a specific time each day, namely at ZT0, ZT4, ZT8, ZT12, ZT16, or ZT20. The animals were euthanized by conscious decapitation $4 \mathrm{~h}$ after the third gavage on day 45 . Using this protocol, it has been shown that serum alcohol level at sacrifice is not affected by time, with mean serum alcohol level per ZT not exceeding $1 \mathrm{~mm}$ [22]. Proximal colon was collected every $4 \mathrm{~h} /$ cohort across the diurnal cycle, starting at ZT0. Tissue was then formalinfixed and paraffin-embedded before sectioning at 3-4 $\mu \mathrm{m}$.

\section{Immunohistochemistry}

Immunoperoxidase (IP) and immunofluorescence (IF) staining were performed according to an established protocol [23]. Tissue samples were first deparaffinized and rehydrated in xylene and $\mathrm{EtOH}$ gradients. Slides were then heated in 5\% antigen retrieval (S1699, DAKO) and probed with primary antibodies. Antibodies were used at the following concentrations: CD3 1:100 (ab5690, Abcam), T-bet 1:400 (ab91109, Abcam), and IF: Foxp3 1:200 (145773-82, eBioscience). Biotinylated secondary antibodies were used in a 1:200 concentration, goat anti-rabbit (BA-1000, Vector Laboratories), horse anti-mouse (BA-2000, Vector Laboratories), IF: donkey anti-rat (Alexa Fluor 488, Thermofisher). Sections were then incubated in ABC complex (PK-6100, Vector Laboratories) for 75 min. Diaminobenzidine (D5637-1G, Sigma-Aldrich) was used for the color development of IP stains. Slides were stained with DAPI (D3571, ThermoFisher) before mounting using aqueous media (Fluoromount, Sigma-Aldrich).

\section{Microscopic Analysis}

IP images were obtained using a light microscope (Leica DMR; $\times 63.3$. IF images were obtained using a fluorescence microscope (Olympus IX81; $\times 40$. For each of the 60 samples stained, 7 blinded and randomly chosen fields were imaged per antibody. Proximal colon tissue has an endogenously high lipid content which may cause autofluorescence; caution was taken to ensure only positive cells were counted. Identified FITC green-positive cells were overlaid with DAPI (blue) and CY3 (red) channels in order to eliminate autofluorescence and nonspecific false-positives. Cells that fluo- resced in the green and red channels were determined to be a product of autofluorescence, as no red-conjugated antibodies were used. Manual counting was possible due to the small number of positive cells for all cell markers. Cell size varied, so traditional counting software would have been less accurate. Manual counting was performed by a single independent blinded investigator, counting all samples twice and taking the average. To avoid any potential scoring bias, a subset of images was scored by another blinded independent investigator; the results were determined to be uniform (online suppl. Fig. A; see www.karger.com/ doi/10.1159/000507124). The surface area of the tissue was determined using BioPix software (BioPix $\mathrm{AB}$ ) counting only the fluoresced tissue area and discarding the black background; this resulted in the number of positive cells $/ \mathrm{mm}^{2}$.

\section{Statistical Analysis}

For all analyses, statistics were performed on mean positive cells divided by mean tissue area (proximal colon lamina propria). First, we evaluated average cells/tissue area over 6 ZTs $(24 \mathrm{~h})$ for the control and alcohol groups $(n=30)$ using unpaired Student's $t$ test. Next, we evaluated the effect of treatment time point in the light (rest phase; mean of ZT0, ZT4, and ZT8) and in the dark (activity phase; mean of ZT12, ZT16, and ZT20) $(n=15)$ using unpaired Student's $t$ test and two-way ANOVA. It has recently been shown that at the start of both murine and human activity cycles, leukocyte trafficking in tissues is at its greatest [15]; it thus stands to reason that immune cells may be present mostly in the gut tissue at this time. A priori, we decided that the onset of the activity phase (dark; ZT12) would be a valuable point for analysis (by unpaired Student $t$ test). In addition to finding mean positive cells at ZT12, we analyzed mean positive cells at each ZT binge time point $(n=5)$ using one-way ANOVA for the control and alcohol-treatment groups. Finally, we analyzed differences in average cells/tissue area between each control and alcohol binge ZT $(n=5)$ using two-way ANOVA and a priori post hoc comparison (unpaired Student's $t$ test). All statistical analyses were performed with GraphPad Prism (GraphPad software), and SPSS (IBM) ( $p<0.05$ was considered significant).

\section{Results}

\section{Alcohol Effect on $\mathrm{CD}^{+} \mathrm{T}$ Cells in the Colon}

$\mathrm{CD} 3$ is the T cell receptor present on the surface of most $\mathrm{T}$ cells. There appeared to be a diurnal fluctuation in the abundance of $\mathrm{CD}^{+}$cells in the colon of control

Fig. 1. $\mathrm{CD}^{+} \mathrm{T}$ cells in the proximal colon. Proximal colon samples of mice treated with $\mathrm{PBS}$ or alcohol binge at the same time point each day stained for CD3, the T cell receptor, using IP staining. a Mean $\mathrm{CD}^{+} \mathrm{T}$ cells counts of all ZTs collected over $24 \mathrm{~h}(n=30)$ unpaired Student's $t$ test, $p=$ ns. b Mean $\mathrm{CD}^{+} \mathrm{T}$ cells following treatment during active/rest phases $(n=15)$ two-way ANOVA and a priori post hoc comparison via Student's $t$ test, $p=$ ns. c Mean $\mathrm{CD}^{+} \mathrm{T}$ cells at each ZT. PBS treatment (left), alcohol treatment (right) $(n=5)$ analyzed via one-way ANOVA, $p=n s ;$ a priori post hoc comparison via unpaired Student's $t$ test, ${ }^{* *} p<0.008$. d Representative photomicrographs depicting $\mathrm{CD}^{+} \mathrm{T}$ cells at ZT4, ZT12, and ZT16. e Mean CD3 ${ }^{+} \mathrm{T}$ cells counts at separate ZTs for both PBS and alcohol groups $(n=5)$ analyzed via two-way ANOVA and a priori post hoc comparison via unpaired Student's $t$ test, $p=$ ns. ZT, Zeitgeber time.

(For figure see next page.) 

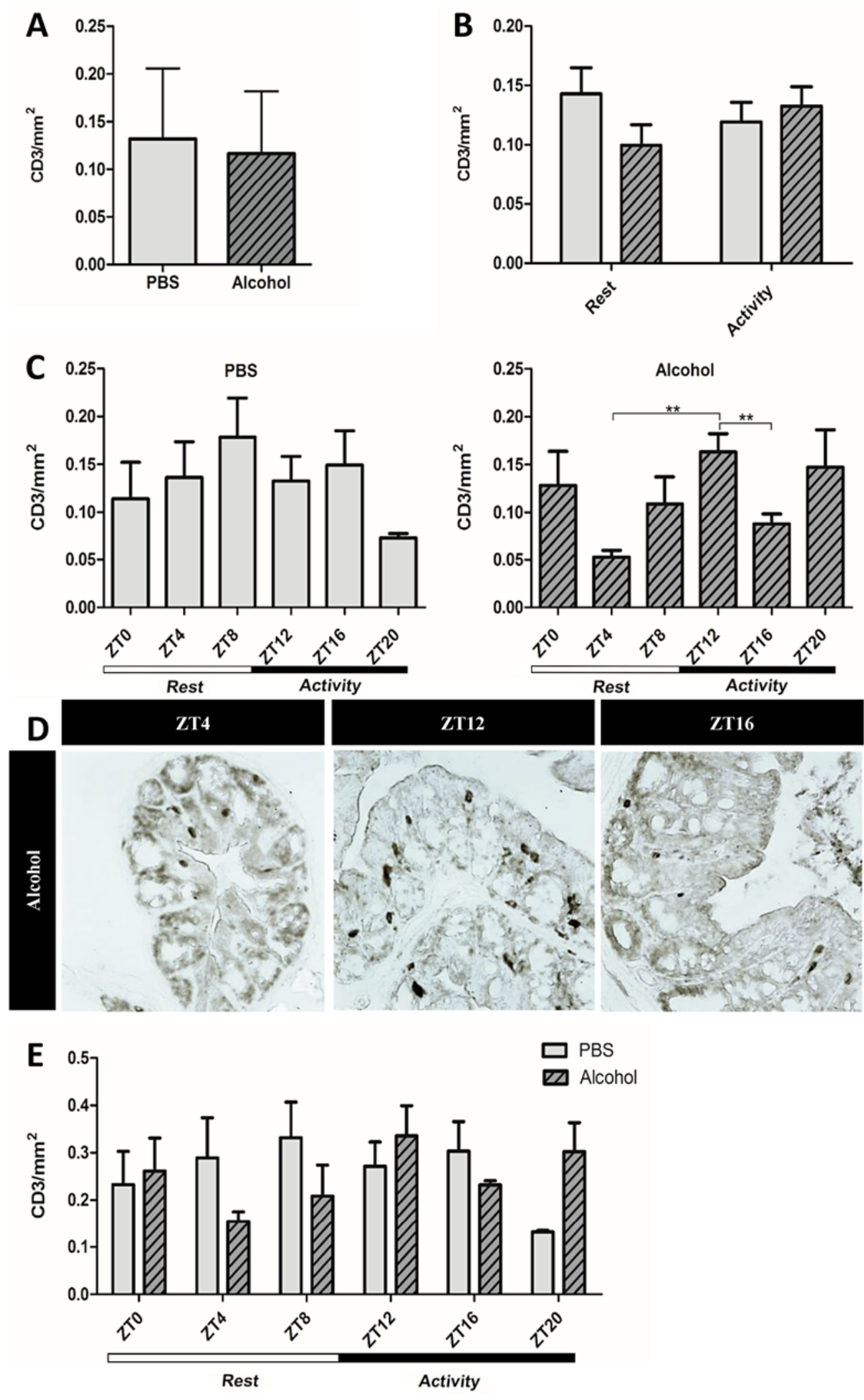


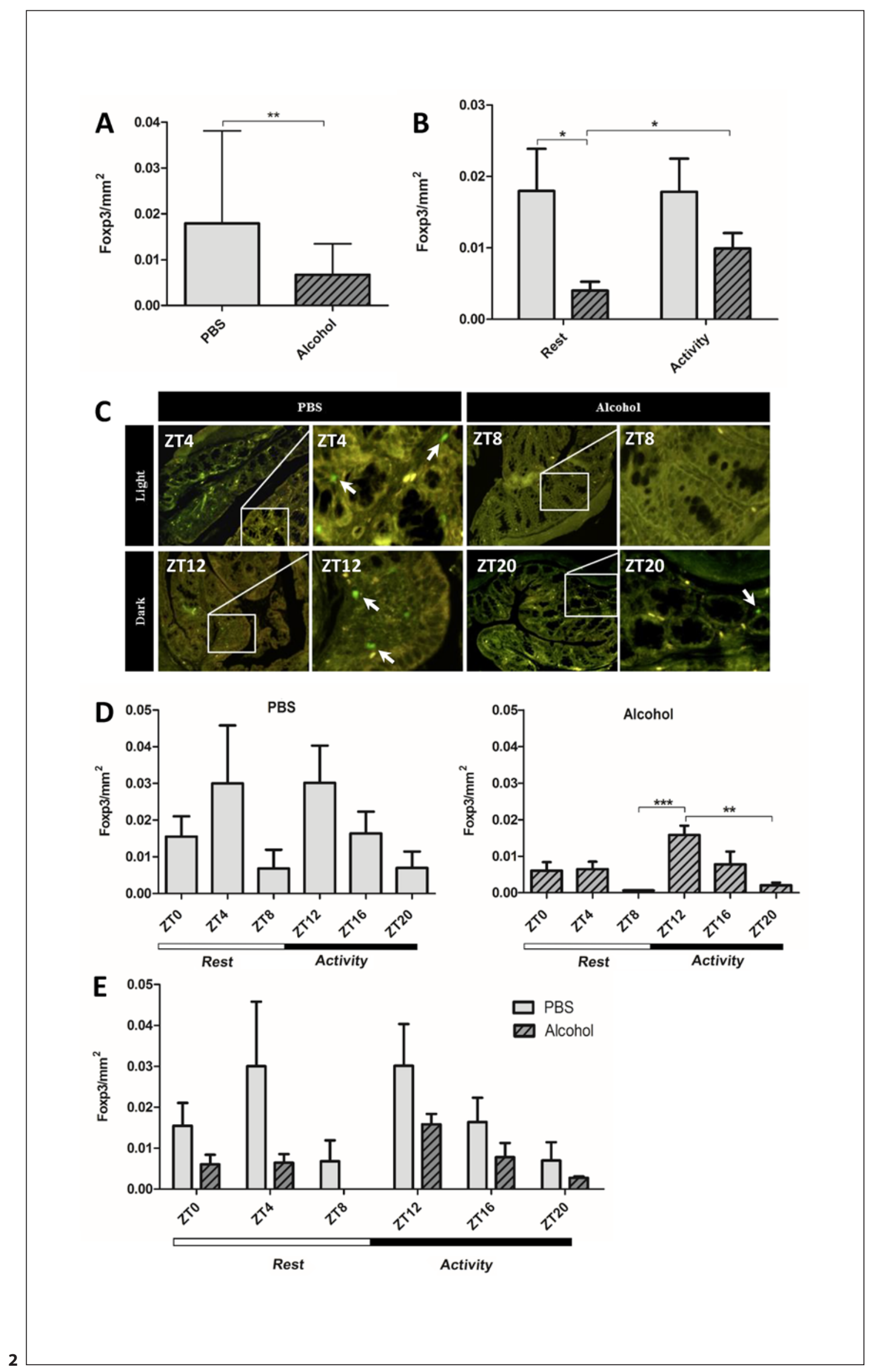

(For legend see next page.) 
animals, but this failed to reach statistical significance. Neither the alcohol treatment nor the period of treatment (rest vs. active phase) affected the overall abundance of mucosal $\mathrm{CD}^{+}$cells in the colon (Fig. 1a-b). However, in the alcohol-treated mice, $\mathrm{CD}^{+}{ }^{+} \mathrm{T}$ cells at $\mathrm{ZT} 12$ were significantly more abundant than at ZT4 and ZT16 (Fig. 1cd). Overall, two-way ANOVA did not reveal any significant effects of alcohol or time of day (Fig. 1e) on the total number of $\mathrm{CD}^{+} \mathrm{T}$ cells in the colon.

\section{Alcohol Reduced Foxp $3^{+}$Tregs in the Colon in a \\ Time-Dependent Manner}

Mucosal inflammation in colon cancer is characterized by a proinflammatory state, with a higher ratio of proinflammatory T cells to Foxp $3^{+}$Tregs [24, 9, 25-27]. Thus, we stained for Foxp3, the transcription factor in Tregs. Alcohol treatment significantly reduced the average number of Foxp $3^{+}$Tregs in the colon (Fig. 2a). Interestingly, the alcohol versus control effect on the abundance of Foxp $3^{+}$cells was significant if alcohol was given during the rest phase (Fig. 2b). There were also significantly fewer Foxp $3^{+}$Tregs present in the colon of mice treated with alcohol during the rest phase than during the active phase (Fig. 2b-c). While the diurnal fluctuations in Foxp $3^{+}$cells per individual ZT showed no significant effect of time (Fig. 2d, left), the time effect was significant in the alcoholtreated group (Fig. 2d, right), indicating a diurnal variation in alcohol's effect on the abundance of colonic Foxp $3^{+}$ cells. Overall, there was a significant effect of time as well as alcohol treatment, with alcohol dramatically reducing the number of Foxp $3^{+}$Tregs present in the colon (Fig. 2e).

\section{T-bet ${ }^{+}$Th1 Cells in the Colon in Response to Alcohol}

We next stained for T-box transcription factor ( $\mathrm{T}$ bet $^{+}$), a transcription factor of Th1 cells. Alcohol did not impact the average number of T-bet ${ }^{+}$cells (Fig. 3a) over $24 \mathrm{~h}$. While the effect of alcohol on T-bet ${ }^{+}$cell count did

Fig. 2. Foxp $3^{+}$Tregs in the proximal colon. Proximal colon samples of mice treated with PBS or alcohol binge at the same time point each day stained for Foxp3 using IF staining. a Mean Foxp $3^{+}$ cell counts at all ZTs over $24 \mathrm{~h}(n=30)$, unpaired Student's $t$ test, ** $p<0.01$. b Mean Foxp $3^{+}$Tregs following treatment during active/rest phases $(n=15)$ two-way ANOVA, alcohol binge $p<0.01$, binge time $p=n s$, interaction $p=n s$; a priori post hoc comparison via Student's $t$ test, ${ }^{*} p<0.03$. c Representative photomicrographs depicting Foxp $3^{+}$Tregs during active/rest phases. d Mean Foxp $3^{+}$ Tregs at each ZT $(n=5)$. Left: PBS; one-way ANOVA, $p=n s ;$ a priori post hoc comparison via unpaired Student's $t$ test, $p=$ ns. Right: alcohol; one-way ANOVA, $p<0.001$; a priori post hoc comparison via unpaired Student's $t$ test, ${ }^{* *} p<0.01$, ${ }^{* * *} p<0.001$. e Mean Foxp $3^{+}$Tregs cell count at separate ZTs for both PBS and alcohol groups $(n=5)$; two-way ANOVA, alcohol binge $p<0.008$, binge time $p<0.05$, interaction $p=\mathrm{ns}$; a priori post hoc comparison via unpaired Student's $t$ test, $p=$ ns. not appear to be different between the rest phase (ZT0, ZT4, and ZT8) and active phase (ZT12, ZT16, and ZT20), the control group had less colonic T-bet ${ }^{+}$cells than the alcohol-treated group during the active phase (Fig. 3b). Overall, we did not observe a significant diurnal variation per ZT in either the control or alcohol-binge group (Fig. 3d).

\section{Alcohol Effect on the Proinflammatory State in the Colon Is Time-Dependent}

T-bet ${ }^{+}$cells dominated the $\mathrm{T}$ cell milieu following alcohol use, indicating stimulation of the Th1 response to the alcohol. To better assess the possible proinflammatory milieu in the colon following alcohol treatment, we assessed the T-bet ${ }^{+}$-to-Foxp $3^{+}$ratio in alcohol-treated and control mice over time; the alcohol treatment was found to triple this ratio compared to the PBS treatment. (Fig. 4a, left). Interestingly, this ratio was highest when alcohol was administered during the light (rest) phase (Fig. 4a, right). Overall, we observed that the abundance of colonic Tregs was downregulated by alcohol, particularly when alcohol was given during the rest phase. With their numbers depleted by the alcohol, the Foxp $3^{+}$Tregs may no longer have been available to downregulate Th1 cell differentiation in this environment. Overall, these data highlight the immune-modulating effects of the alcohol treatment and its interaction with time in promoting proinflammatory consequences in the colon.

\section{Discussion}

Alcohol-induced CRC has also been shown to be associated with a proinflammatory state in the colon. This study is the first to address the role of time in the mucosal immune response to alcohol in the colon. We describe the abundance of total and subset $\mathrm{T}$ cell populations in the colon of mice following alcohol treatment at 6 different time points throughout the day. Our findings revealed that alcohol intake, particularly during the rest phase, is associated with a proinflammatory $\mathrm{T}$ cell milieu in the mouse colon. The impact of alcohol on mucosal immunity composition in the colon, the principal site of alcohol-induced leaky gut and dysbiosis [24, 26, 27], has remained understudied. In the small intestine, chronic alcohol intake has been shown to result in dysregulated immune cell function, more so than a reduction in $\mathrm{T}$ cell numbers $[28,29]$. Although we found a slight reduction in the number of $\mathrm{CD}^{+} \mathrm{T}$ cells upon treatment with alcohol, this did not reach significance. In the jejunum of macaques, chronic alcohol has been shown to reduce $\mathrm{CD} 4^{+}$ $\mathrm{T}$ cells as well as effector memory $\mathrm{T}$ cells [28]. While chronic alcohol may have an immunosuppressive effect in the gut, in our model of acute alcohol binge, the ratio 

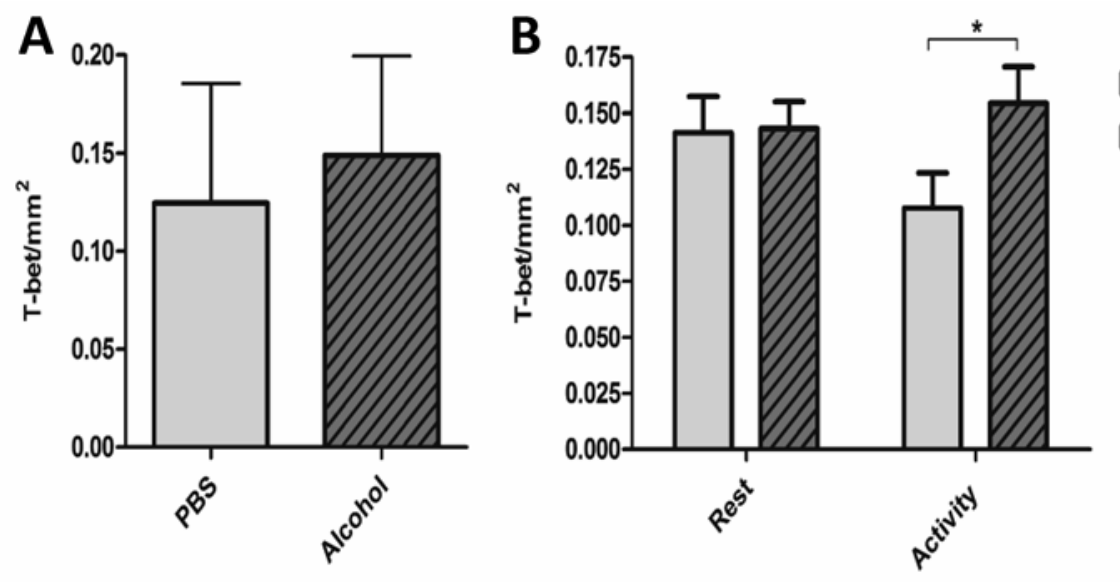

PBS

Alcohol
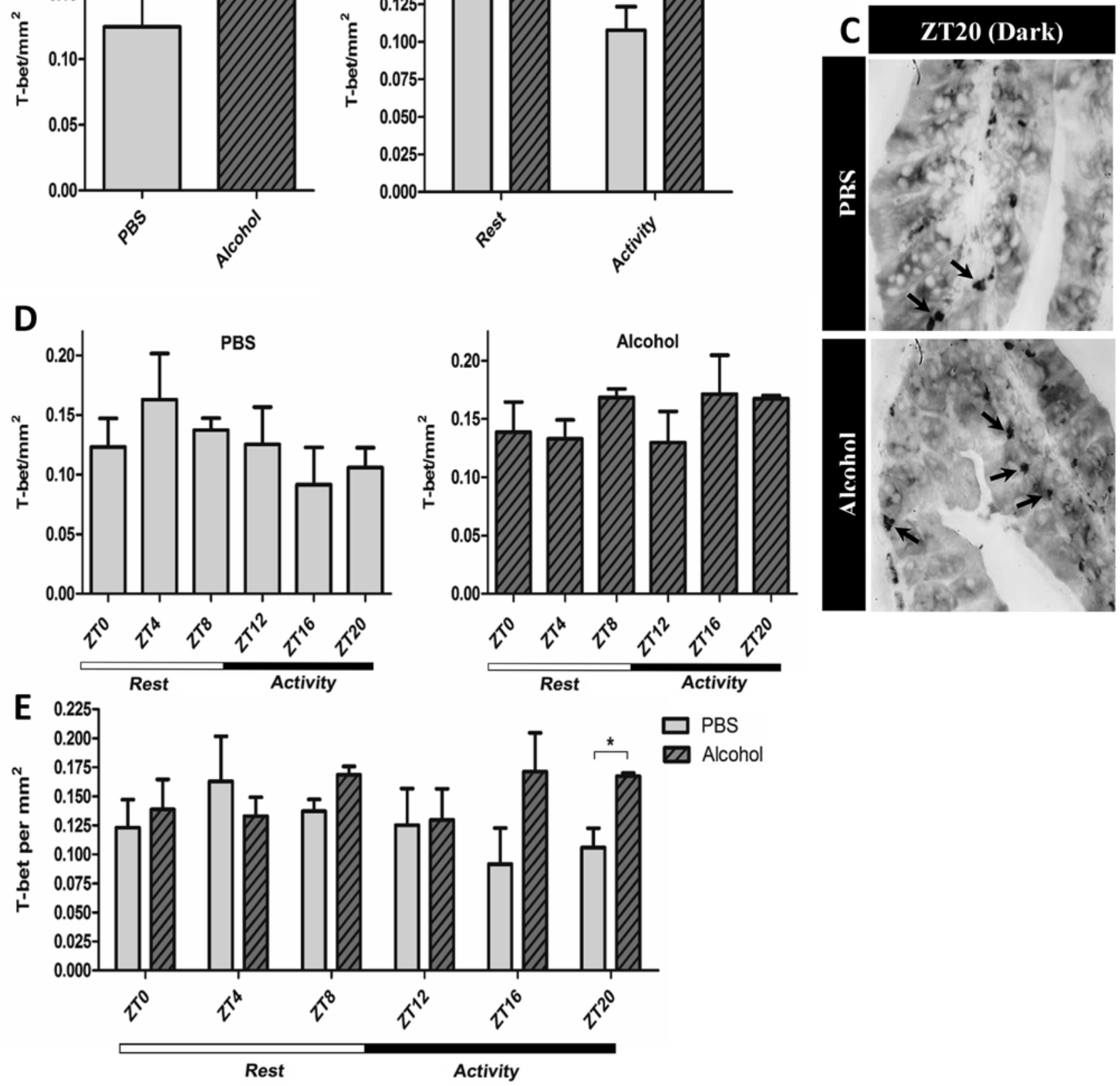

Fig. 3. T-bet $^{+}$Th1 cells in the proximal colon. Proximal colon samples of mice treated with PBS or alcohol binge stained for Tbet using IP staining. a Mean T-bet ${ }^{+}$Th 1 cell count of all ZTs collected over $24 \mathrm{~h}(n=30)$; Student's unpaired $t$ test, $p=$ ns. b Mean T-bet ${ }^{+}$Th1 cells during active/rest phases $(n=15)$, two-way ANOVA, $p=n s$; a priori post hoc comparison via unpaired Student's $t$ test, ${ }^{*} p<0.05$. c Representative photomicrographs depict- ing T-bet ${ }^{+}$Th1 cells at ZT20. d Mean T-bet ${ }^{+}$Th1 cells at each ZT $(n=5)$; one-way ANOVA, $p=n s$; a priori post hoc comparison via Student's $t$ test, $p=$ ns. e Mean T-bet ${ }^{+}$Th 1 cell counts at separate ZTs for both PBS and alcohol groups $(n=5)$; two-way ANO$\mathrm{VA}, p=\mathrm{ns}$; a priori post hoc comparison via unpaired Student's $t$ test, $* p<0.03$. 


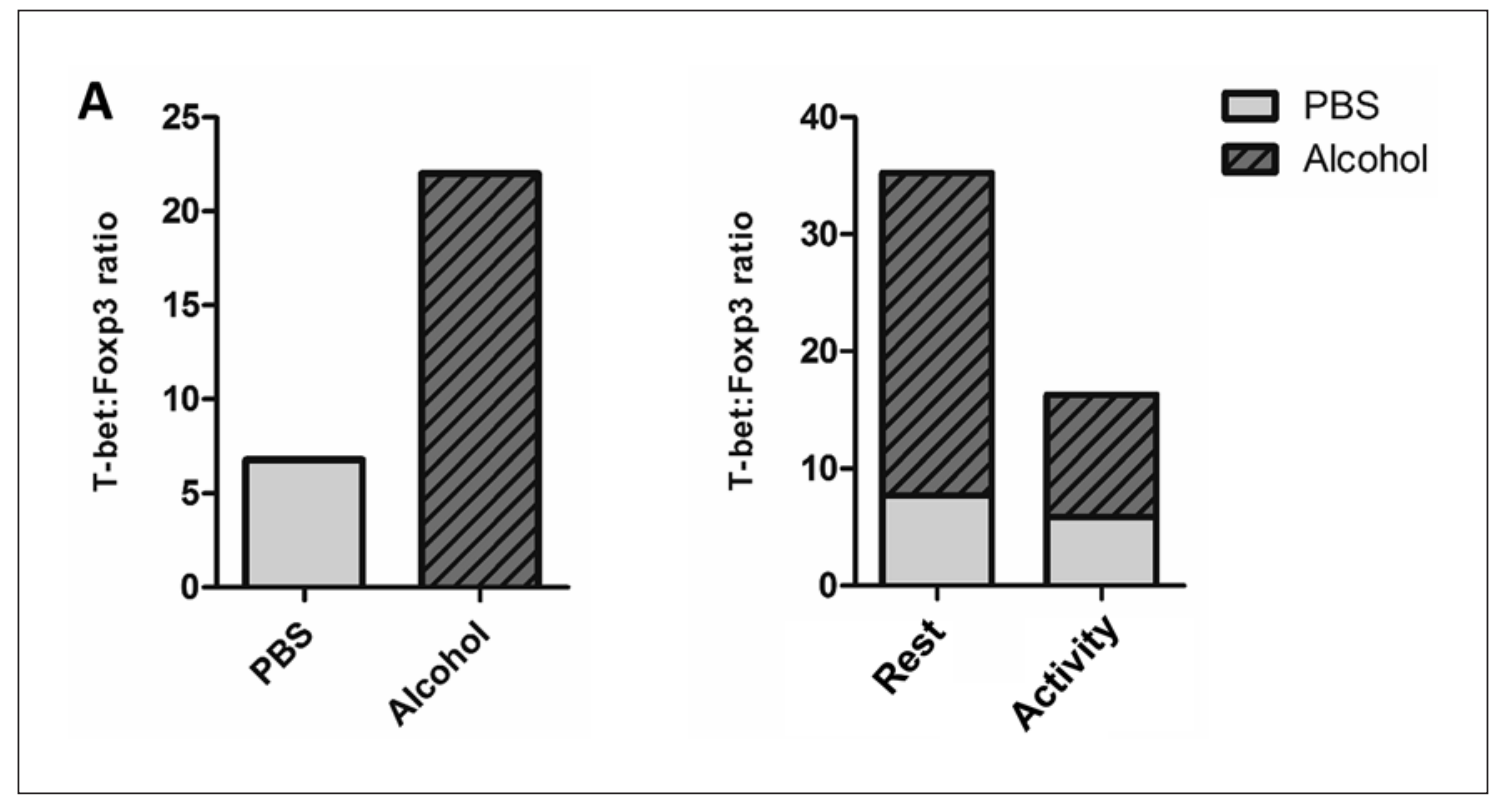

Fig. 4. $\mathrm{T}$-bet ${ }^{+} \mathrm{Th} 1$ cells dominated the $\mathrm{T}$ cell milieu following the alcohol binge. a Left: $\mathrm{T}-\mathrm{bet}{ }^{+} \mathrm{Th} 1$ :Foxp $3^{+}$Treg ratio following PBS and alcohol binge. Right: T-bet ${ }^{+}$Th1:Foxp $3^{+}$Treg ratio following alcohol binge during the rest phase (ZT0/4/8) and active phase (ZT12/16/20).

of colonic Th1 cells-to-Tregs increased. Alcohol binge induced a significant reduction in Foxp $3^{+}$Tregs, which are known to produce the immunosuppressive cytokine IL10. IL-10 decreases the production of inflammatory cytokines such as TNF- $\alpha$, IL-1, and IL-6 [30].

A reduction in Foxp3+ Tregs during the acute phase of alcohol treatment may have resulted in an immunedysregulated state in which inflammation could be primed. Supporting this, we found that T-bet ${ }^{+}$Th1 cells dominated the $\mathrm{CD}^{+} \mathrm{T}$ cell subset population following alcohol treatment. Upon differentiation, immune cells leave the mesenteric lymph nodes and traffic to the intestine via a gradient of chemokines released by damaged epithelial cells and resident immune cells [31]. While alcohol is known to affect immune cell differentiation [32, 33], the diurnal variation of immune cell abundance in the colon that we saw in response to the alcohol is possibly not explained by differentiation alone. Consistent with this, we recently reported that numbers of effector $\mathrm{T}$ cells and Tregs in the mesenteric lymph nodes did not differ in the alcohol-treated mice fed in the active phase and the rest phase [14]. Leukocyte levels in peripheral tissues usually peak at the onset of the active phase (i.e., the dark phase for mice) $[17,19]$. Our data suggest that the effect of alcohol on $\mathrm{T}$ cell trafficking to the colon could be distinct in these 2 phases, with alcohol consumed during the rest phase resulting in a proinflammatory $\mathrm{T}$ cell milieu in the colon. In our study, we did not find a higher average number of leukocytes in the colon tissue present during the active phase than during the rest phase. However, in alcohol-treated mice, colonic Foxp $3^{+}$Tregs in the active phase were more abundant than in the rest phase. The Tbet $^{+}$Th1 numbers increased significantly in the colon of mice treated with alcohol during the active phase. Overall, the abundance of colonic Foxp $3^{+}$Tregs was significantly affected by both alcohol treatment and the time of treatment. How the time point of alcohol intake affects colonic immune cell abundance, and whether trafficking, rather than $\mathrm{T}$ cell proliferation and differentiation, accounts for the diurnal effect of alcohol on colonic leukocytes needs to be further established [34, 12]. In summary, our data suggest that the composition of immune cell subsets in the colon tissue in response to alcohol could be time-dependent. Following alcohol treatment, we observed a predominant Th1 lymphocyte population with reduced Treg numbers. This reduction in Tregs leads to a significant increase in the Th1 cell-to-Treg ratio, which tips the balance towards a more proinflammatory milieu. This effect was time-dependent, with Tregs most readily reduced when alcohol was administered during the rest phase. The time variation of the colon immune cell milieu in response to alcohol may partly explain the promoting effect of circadian rhythm disruption on alcohol-induced colon inflammation and CRC.

\section{Statement of Ethics}

This study was reviewed and approved by the Institutional Review Board and all animal experiments were conducted and approved by the Institutional Animal Care and Use Committee of Rush University Medical Center. 


\section{Disclosure Statement}

None of the authors has any potential conflicts (financial, professional, or personal) related to the manuscript to disclose.

\section{Funding Sources}

F.B. is supported by NIH/NIAAA: AA025387 as well as Rush Translational Sciences Consortium/Swim Across America Organization grant. C.B.F. is supported by NIH/NIAAA: AA026801.

\section{Author Contributions}

E.L.G. performed the experiments, analyzed the data, and drafted the results. A.R.A. helped with the preparation of figures and edited the manuscript. C.B.F. and R.M.V. assisted in the study design and supervision. F.B. was involved in the study design and data interpretation and wrote the manuscript. All authors read and approved the final manuscript.

\section{References}

1 Fedirko V, Tramacere I, Bagnardi V, Rota M, Scotti L, Islami F, et al. Alcohol drinking and colorectal cancer risk: an overall and dose-response meta-analysis of published studies. Ann Oncol. 2011 Sep;22(9):1958-72.

2 Zhu JZ, Wang YM, Zhou QY, Zhu KF, Yu CH, Li YM. Systematic review with meta-analysis: alcohol consumption and the risk of colorectal adenoma. Aliment Pharmacol Ther. 2014 Aug; 40(4):325-37.

3 Wimberly AL, Forsyth CB, Khan MW, Pemberton A, Khazaie K, Keshavarzian A. Ethanolinduced mast cell-mediated inflammation leads to increased susceptibility of intestinal tumorigenesis in the APC $\Delta 468 \mathrm{~min}$ mouse model of colon cancer. Alcohol Clin Exp Res. 2013 Jan;37 Suppl 1:E199-208.

4 Shukla PK, Chaudhry KK, Mir H, Gangwar R, Yadav N, Manda B, et al. Chronic ethanol feeding promotes azoxymethane and dextran sulfate sodium-induced colonic tumorigenesis potentially by enhancing mucosal inflammation. BMC Cancer. 2016 Mar;16(1):189.

5 Bishehsari F, Magno E, Swanson G, Desai V, Voigt RM, Forsyth CB, et al. Alcohol and GutDerived Inflammation. Alcohol Res. 2017; 38(2):163-71.

6 Salama P, Phillips M, Grieu F, Morris M, Zeps $\mathrm{N}$, Joseph D, et al. Tumor-infiltrating FOXP3+ $\mathrm{T}$ regulatory cells show strong prognostic significance in colorectal cancer. J Clin Oncol. 2009 Jan;27(2):186-92.

7 Correale P, Rotundo MS, Del Vecchio MT, Remondo C, Migali C, Ginanneschi C, et al. Regulatory (FoxP3+) T-cell tumor infiltration is a favorable prognostic factor in advanced colon cancer patients undergoing chemo or chemoimmunotherapy. J Immunother. 2010 May; 33(4):435-41.

8 Nguyen AV, Wu YY, Liu Q, Wang D, Nguyen $\mathrm{S}$, Loh R, et al. STAT3 in epithelial cells regulates inflammation and tumor progression to malignant state in colon. Neoplasia. $2013 \mathrm{Sep}$; 15(9):998-1008.

9 Leonel AJ, Alvarez-Leite JI. Butyrate: implications for intestinal function. Curr Opin Clin Nutr Metab Care. 2012 Sep;15(5):474-9.

10 Hoogerwerf WA, Hellmich HL, Cornélissen G, Halberg F, Shahinian VB, Bostwick J, et al. Clock gene expression in the murine gastrointestinal tract: endogenous rhythmicity and effects of a feeding regimen. Gastroenterology. 2007 Oct;133(4):1250-60.

11 Rossi M, Mirbagheri SE, Keshavarzian A, Bishehsari F. Nutraceuticals in colorectal can- cer: A mechanistic approach. Eur J Pharmacol. 2018 Aug;833:396-402.

12 Summa KC, Voigt RM, Forsyth CB, Shaikh M, Cavanaugh K, Tang Y, et al. Disruption of the Circadian Clock in Mice Increases Intestinal Permeability and Promotes Alcohol-Induced Hepatic Pathology and Inflammation. PLoS One. 2013 Jun;8(6):e67102.

13 Voigt RM, Forsyth CB, Keshavarzian A. Circadian disruption: potential implications in inflammatory and metabolic diseases associated with alcohol. Alcohol Res. 2013;35(1):87-96.

14 Bishehsari F, Engen PA, Voigt RM, Swanson G, Shaikh M, Wilber S, et al. Abnormal Eating Patterns Cause Circadian Disruption and Promote Alcohol-Associated Colon Carcinogenesis. Cell Mol Gastroenterol Hepatol. 2020; 9(2):219-37.

15 Janakiram NB, Rao CV. The role of inflammation in colon cancer. Adv Exp Med Biol. 2014; 816:25-52.

16 De Simone V, Pallone F, Monteleone G, Stolfi C. Role of TH17 cytokines in the control of colorectal cancer. OncoImmunology. 2013 Dec;2(12):e26617.

17 Sakaguchi S, Miyara M, Costantino CM, Hafler DA. FOXP3+ regulatory T cells in the human immune system. Nat Rev Immunol. 2010 Jul;10(7):490-500.

18 Moossavi S, Bishehsari F. Inflammation in sporadic colorectal cancer. Arch Iran Med. 2012 Mar; 15(3):166-70.

19 Hanyuda A, Ogino S, Qian ZR, Nishihara R, Song M, Mima K, et al. Body mass index and risk of colorectal cancer according to tumor lymphocytic infiltrate. Int J Cancer. 2016 Aug; 139(4):854-68.

20 Bishehsari F, Zhang L, Voigt RM, Maltby N, Semsarieh B, Zorub E, et al. Alcohol Effects on Colon Epithelium are Time-Dependent. Alcohol Clin Exp Res. 2019 Sep;43(9):1898-908.

21 Bertola A, Mathews S, Ki SH, Wang H, Gao B. Mouse model of chronic and binge ethanol feeding (the NIAAA model). Nat Protoc. 2013 Mar;8(3):627-37.

22 Voigt RM, Forsyth CB, Shaikh M, Zhang L, Raeisi S, Aloman C, et al. Diurnal variations in intestinal barrier integrity and liver pathology in mice: implications for alcohol binge. Am J Physiol Gastrointest Liver Physiol. 2018 Jan; 314(1):G131-41.

23 Engen PA, Dodiya HB, Naqib A, Forsyth CB, Green SJ, Voigt RM, et al. The Potential Role of Gut-Derived Inflammation in Multiple System Atrophy. J Parkinsons Dis. 2017;7(2):331-46.
24 Blatner NR, Mulcahy MF, Dennis KL, Scholtens D, Bentrem DJ, Phillips JD, et al. Expression of ROR $\gamma t$ marks a pathogenic regulatory T cell subset in human colon cancer. Sci Transl Med. 2012 Dec;4(164):164ra159.

25 Ganapathy V, Thangaraju M, Prasad PD, Martin PM, Singh N. Transporters and receptors for short-chain fatty acids as the molecular link between colonic bacteria and the host. Curr Opin Pharmacol. 2013 Dec;13(6):86974.

26 Harrison OJ, Powrie FM. Regulatory T cells and immune tolerance in the intestine. Cold Spring Harb Perspect Biol. 2013 Jul; 5(7):a018341.

27 Tanoue T, Atarashi K, Honda K. Development and maintenance of intestinal regulatory $\mathrm{T}$ cells. Nat Rev Immunol. 2016 May;16(5):295309.

28 Thangaraju M, Cresci GA, Liu K, Ananth S, Gnanaprakasam JP, Browning DD, et al. GPR109A is a G-protein-coupled receptor for the bacterial fermentation product butyrate and functions as a tumor suppressor in colon. Cancer Res. 2009 Apr;69(7):2826-32.

29 Singh N, Gurav A, Sivaprakasam S, Brady E, Padia R, Shi H, et al. Activation of Gpr109a, receptor for niacin and the commensal metabolite butyrate, suppresses colonic inflammation and carcinogenesis. Immunity. 2014 Jan; 40(1):128-39.

30 Stockman NK, Schenkel TC, Brown JN, Duncan AM. Comparison of energy and nutrient intakes among meals and snacks of adolescent males. Prev Med. 2005 Jul;41(1):203-10.

31 Gounaris E, Blatner NR, Dennis K, Magnusson F, Gurish MF, Strom TB, et al. T-regulatory cells shift from a protective anti-inflammatory to a cancer-promoting proinflammatory phenotype in polyposis. Cancer Res. 2009 Jul; 69(13):5490-7.

32 Stokkan KA, Yamazaki S, Tei H, Sakaki Y, Menaker M. Entrainment of the circadian clock in the liver by feeding. Science. 2001 Jan; 291(5503):490-3.

33 Furusawa Y, Obata Y, Fukuda S, Endo TA, Nakato G, Takahashi D, et al. Commensal microbe-derived butyrate induces the differentiation of colonic regulatory T cells. Nature. 2013 Dec;504(7480):446-50.

34 Le Gouvello S, Bastuji-Garin S, Aloulou N, Mansour H, Chaumette MT, Berrehar F, et al. High prevalence of Foxp3 and IL17 in MMRproficient colorectal carcinomas. Gut. 2008 Jun;57(6):772-9. 